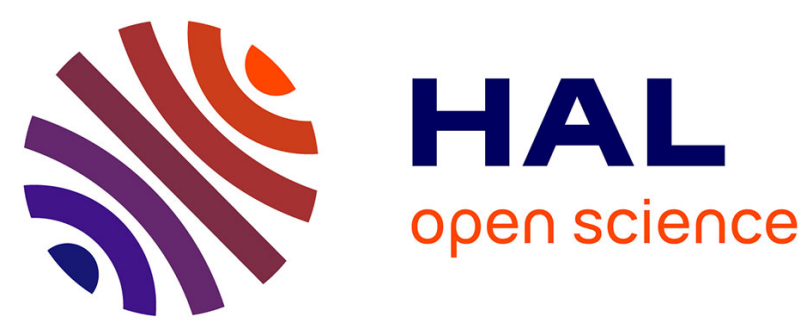

\title{
Influence of hydrogenation on the structural and magnetic properties of compounds based on cerium and crystallizing in the tetragonal CeFeSi-type structure
}

\author{
Bernard Chevalier, Mathieu Pasturel, Jean-Louis Bobet, Olivier Isnard
}

\section{- To cite this version:}

Bernard Chevalier, Mathieu Pasturel, Jean-Louis Bobet, Olivier Isnard. Influence of hydrogenation on the structural and magnetic properties of compounds based on cerium and crystallizing in the tetragonal CeFeSi-type structure. Solid State Communications, 2005, 134 (8), pp.529-533. 10.1016/j.ssc.2005.02.040 . hal-00022430

\section{HAL Id: hal-00022430 \\ https://hal.science/hal-00022430}

Submitted on 7 Apr 2006

HAL is a multi-disciplinary open access archive for the deposit and dissemination of scientific research documents, whether they are published or not. The documents may come from teaching and research institutions in France or abroad, or from public or private research centers.
L'archive ouverte pluridisciplinaire HAL, est destinée au dépôt et à la diffusion de documents scientifiques de niveau recherche, publiés ou non, émanant des établissements d'enseignement et de recherche français ou étrangers, des laboratoires publics ou privés. 


\title{
I nfluence of hydrogenation on the structural and magnetic properties of compounds based on cerium and crystallizing in the tetragonal CeFeSi-type structure
}

\author{
Bernard Chevalier, Mathieu Pasturel, Jean-Louis Bobet, O. I snard
}

\begin{abstract}
:
The hydrogen absorption properties of CeMnGe, CeFeSi and $\mathrm{CeCoX}(\mathrm{X}=\mathrm{Si}$ and $\mathrm{Ge})$ have been investigated. Neutron powder diffraction performed on deuteride CeCoGeD indicates that D-atoms are inserted in the pseudo-tetrahedral interstices $\left[\mathrm{Ce}_{4}\right]$ of the tetragonal CeFeSi-type structure of this compound. Magnetization and electrical resistivity measurements reveal that the hydrogenation of: (i) $\mathrm{CeCoSi}$ and $\mathrm{CeCoGe}$ leads to the transition antiferromagnet $\rightarrow$ spin fluctuation behaviour; (ii) CeMnGe suppress the magnetic ordering of the Ce-moments. These results which suggest a lost of ordered magnetic moment on the Ce site after hydrogenation could result from the chemical effect of hydrogen which prevails over the unit cell expansion effect.
\end{abstract}

Keywords : Magnetically ordered materials ; Valence fluctuations

\section{Introduction}

Recent works devoted to hydrogenation of the intermediate valence compounds based on cerium show that the absorption of hydrogen by these intermetallics induces both an increase of the unit cell volume and a decrease of the cerium valence. For instance, in the CeNiX series with X=Al, Ga, In, Ge and Sn interesting magnetic transitions were evidenced by hydrogenation: (i) a decrease of the Kondo temperature from $600 \mathrm{~K}(\mathrm{CeNiGe})$ to $220 \mathrm{~K}\left(\mathrm{CeNiGeH}_{1.6}\right)$ [1]; (ii) a change from intermediate valence (CeNiGa) to a trivalent state $\left(\mathrm{CeNiGaH}_{1.1}\right)$ for the Ce ions [2]; (iii) the occurrence of ferromagnetic ordering after $\mathrm{H}$-insertion in CeNiIn [3] and [4]; (iv) finally, the sequence Kondo semiconductor $\rightarrow$ antiferromagnet $\rightarrow$ ferromagnet revealed in the $\mathrm{CeNiSnH}_{y}(y=0,1.0$ and 1.8) system [5], [6] and [7]. All these transitions were explained by a decrease of the hybridisation between the $4 \mathrm{f}(\mathrm{Ce})$ electrons and the conduction electrons induced by the hydrogenation [4]. In other words, the hydrogenation acts as the application of a 'negative' pressure on these intermetallics.

Recently, an unusual transition from antiferromagnetic ordering to spin fluctuation behaviour by $\mathrm{H}$ insertion in the ternary germanide CeCoGe which crystallizes in the tetragonal CeFeSi-type structure has been reported [8]. According to the structural properties of the hydride $\mathrm{CeCoGeH}$, it is supposed that the $\mathrm{H}$-atoms are inserted in the $\left[\mathrm{Ce}_{4}\right]$ pseudo-tetrahedral sites of the CeCoGe structure. In this case, the chemical bond between cerium and hydrogen could explain the lost of magnetic moment of cerium in CeCoGeH at low temperature.

In order to verify the hypothesis made on the structural properties of $\mathrm{CeCoGeH}$, neutron powder diffraction on the equivalent deuteride have been performed. The aim of this paper is to discuss on the influence of the hydrogenation on the electrical and magnetic properties of the ternary compounds CeCoSi and $\mathrm{CeMnGe}$. The electronic state of cerium in the compounds crystallizing in the tetragonal CeFeSi-type, should be strongly modified by $\mathrm{H}$-insertion.

\section{Experimental procedures}

All the samples were synthesized by arc-melting a stoichiometric mixture of pure elements (purity above $99.9 \%$ ) in a high purity argon atmosphere. Then, the samples were turned and remelted several times to ensure homogeneity. Annealing was done for one month at $1073 \mathrm{~K}$ by enclosing the sample in evacuated quartz tubes.

Hydrogen absorption experiments were performed using the apparatus described previously [9]. An ingot of an annealed sample was heated under vacuum at $523 \mathrm{~K}$ for $12 \mathrm{~h}$ and then exposed for $48 \mathrm{~h}$ to $5 \mathrm{MPa}$ of hydrogen gas at the same temperature. The amount of $\mathrm{H}$-absorbed was determined volumetrically by monitoring pressure changes in a calibrated volume. The deuteride CeCoGeD was obtained in the same conditions using deuterium gas. 
X-ray powder diffraction with the use of a Philips 1050-diffractometer ( $\mathrm{Cu} \mathrm{K}_{\mathrm{a}}$ radiation) was applied, before and after hydrogenation, for the characterization of the structural type and for the phase identification of the samples. The refinement of the crystal structure of the deuteride CeCoGeD was performed by Rietveld profile refinement using the software Fullprof [10] and the data of powder neutron diffraction collected at room temperature with the D1B diffractometer at Institut Laue Langevin, Grenoble $(\lambda=1.287 \mathrm{~A})$.

Magnetization measurements were performed using a Superconducting quantum interference device (SQUID) magnetometer in the temperature range 1.8-370 $\mathrm{K}$ and applied fields up to $5 \mathrm{~T}$. Electrical resistivity was determined above $4.2 \mathrm{~K}$ on a polycrystalline sample using standard dc four-probe measurements. For this investigation, the hydride (CeCoSiH) was compacted at room temperature (compactness $\cong 80 \%$ ) in order to form a pellet (diameter $=6 \mathrm{~mm}$ and thickness $=3 \mathrm{~mm}$ ) and then heated for $48 \mathrm{~h}$ at $523 \mathrm{~K}$ under a pressure ( $5 \mathrm{MPa}$ ) of hydrogen.

\section{Results and discussion}

Under the experimental conditions described previously, the compounds $\mathrm{CeCoX}(\mathrm{X}=\mathrm{Si}, \mathrm{Ge}), \mathrm{CeFeSi}$ and $\mathrm{CeMnGe}$ absorb hydrogen. The amount of $\mathrm{H}$-atom inserted is close to 1.0 per formula unit (Table 1 ). All the hydrides formed are stable after the synthesis and do not release any significant amounts of hydrogen under normal conditions. Moreover, the $\mathrm{H}$-absorption induces a decrepitation in small particles of the starting ingot. The crystal structure of these ternary compounds and their hydrides adopt the same tetragonal space group $P 4 / \mathrm{nmm}$ (no. 129) but the hydrogenation causes a pronounced anisotropic expansion of the unit cell (Table 1); the a-parameter decreases whereas the $c$-parameter increases strongly (for instance, $\Delta c / c$ ratio is, respectively, 8 and $15 \%$ after $\mathrm{H}$-insertion into the CeMnGe and CeFeSi lattices). In other words, hydrogenation of these compounds involves an expansion of the unit cell volume.

\subsection{Crystal structure of CeCoGeD}

The neutron diffraction patterns collected at room temperature for CeCoGe and CeCoGeD reveal the presence of $\mathrm{CeCO}_{2} \mathrm{Ge}_{2}$ ( $\cong 6 \%$ in mass) as impurity. These two patterns present nuclear Bragg peaks associated with the tetragonal CeFeSi-type structure (space group $P 4 / \mathrm{nmm}$, no. 129). Results of the refinement of the crystal structure of CeCoGe and its deuteride are given in Table 2. In the refinement of the CeCoGe diffraction pattern, attempt have been made to refine the Co and Ge occupancies, but according to the results given in Table 2, a full ordering of the Co and Ge atoms has been obtained. Since, the same sample has been used to produce the CeCoGeD deuteride, the metallic site occupancies were kept to $100 \%$ for the investigation of this sample. The atomic position parameters determined for CeCoGe are in agreement with those reported previously [14]. It is interesting to note that the deuterium atoms occupy a single crystallographic site on the Wyckoff position $2 \mathrm{~b}$. D-atoms fill the pseudo-tetrahedral interstices $\left[\mathrm{Ce}_{4}\right]$ (Fig. 1). The structure of CeCoGeD can be described as a stacking along the c-axis of two layers formed by $\left[\mathrm{Ce}_{4} \mathrm{CO}_{4}\right]$ antiprisms inserting $\mathrm{Ge}$-atoms and separated by one layer of $\left[\mathrm{Ce}_{4}\right]$ pseudotetrahedral units inserting $D$-atoms. The bonding distances $C e-D$ are all equal to $2.43(1) \AA$. Another interesting feature concerns the evolution of the $\mathrm{Ce}-\mathrm{Ce}$ interatomic distances after deuterium insertion: these forming the $\left[\mathrm{Ce}_{4} \mathrm{Co}_{4}\right]$ antiprisms decreases from 4.190(1) to 4.044(1) A whereas the four other existing in the $\left[\mathrm{Ce}_{4}\right]$ pseudo-tetrahedral increase from $3.87(1)$ to $3.93(1) \AA$

$\mathrm{H}$-insertion into CeCoGe affects strongly the atomic coordinate of Ge-atom: $\mathrm{z}_{\mathrm{Ge}}$ varies from 0.1924(8) to $0.1632(8)$ (Table 2). In other words, Ge-atom shifts along $c$-axis in the $\left[\mathrm{Ce}_{4} \mathrm{Co}_{4}\right]$ antiprisms. This induces a decrease of the Co-Ge interatomic distances from $2.477(1)$ to $2.385(1) \AA$.

\subsection{Transition from antiferromagnet to spin fluctuation behaviour induced by hydrogenation of $\mathrm{CeCoSi}$}

Recent investigation of CeCoSi and $\mathrm{CeCoSiH}$ by magnetic susceptibility $\chi_{\mathrm{m}}$ measurements reveals [13]: (i) that CeCoSi exhibits an antiferromagnetic transition at $T_{N}=8.8(2) \mathrm{K}$; (ii) on the contrary, the curve $X_{\mathrm{m}}=f(T)$ relative to the hydride CeCoSiH shows a broad peak around $65(3) \mathrm{K}$ such as that of $\mathrm{CeSn}_{3}$ which is a typical valence fluctuation material [15]. Using the model offered by Lawrence [15], the spin fluctuation temperature resulting from the hybridisation between $4 \mathrm{f}(\mathrm{Ce})$ and conduction electrons was estimated to $T_{\mathrm{sf}}=130(5) \mathrm{K}$. This analysis suggests that the hydrogenation of CeCoSi leads to the transition antiferromagnet $\rightarrow$ spin fluctuation behavior.

This assumption is confirmed by the temperature dependence of the reduced electrical resistivity of $\mathrm{CeCoSi}$ and its hydride (Fig. 2). For CeCoSi, the resistivity exhibits a sharp decrease at $8.8(2) \mathrm{K}$ which is associated to an onset of a coherent diffusion of the conduction electrons and agrees with the occurrence of the antiferromagnetic ordering evidenced by magnetization measurements. A different feature appears 
for $\mathrm{CeCoSiH}$. In this case, the curve $\rho(T) / \rho(270 \mathrm{~K})=f(T)$ shows a large maximum around $50(5) \mathrm{K}$, associated with a steep decrease below this temperature. This variation is very similar to that observed in $\mathrm{Ce}\left(\mathrm{Pt}_{1-x} \mathrm{Ni}_{x}\right) \mathrm{Si}(0.5 \leq x \leq 1)$ intermediate valence system [16]. It is noted that the temperature $(50(5) \mathrm{K})$ where the $\rho(T) / \rho(270 \mathrm{~K})=f(T)$ curve goes through a maximum is close to the maximum of the $X_{\mathrm{m}}=f(T)$ one (i.e. $65(3) \mathrm{K}$ ). This agrees with the interpretation that the resistivity peak is due to a spin-scattering mechanism in the intermediate valence regime [16].

\subsection{Suppression of the Ce-magnetic ordering by hydrogenation of CeMnGe}

Fig. 3 presents the temperature dependence of the magnetization $M$ for CeMnGe and its hydride in the temperature range $2-340 \mathrm{~K}$ and in an applied magnetic field $\mu_{0} H=0.1 \mathrm{~T}$. For CeMnGe, two peaks characteristics of the occurrence of antiferromagnetic ordering are clearly distinguished at $T_{\mathrm{N} 1}=313(2) \mathrm{K}$ and $T_{\mathrm{N} 2}=41(2) \mathrm{K}$. According to the investigation of the antiferromagnetic structures of CeMnGe reported previously [11], the peak at $T_{\mathrm{N} 1}$ evidences the antiferromagnetic ordering of the $\mathrm{Mn}$-sublattice whereas $T_{\mathrm{N} 2}$ corresponds to the magnetic ordering of the Ce-moments which are coupled ferromagnetically within the $\left[\mathrm{Ce}_{4}\right]$ pseudo-tetrahedral units and antiferromagnetically within the $\left[\mathrm{Ce}_{4} \mathrm{Mn}_{4}\right]$ antiprisms. It is noteworthy that magnetic orderings which occurs on both $\mathrm{Mn}$ and Ce sublattices were not detected previously by magnetization measurements [11].

Between $T_{\mathrm{N} 1}$ and $T_{\mathrm{N} 2}$, the magnetization of CeMnGe increases almost linearly with the applied magnetic field (Fig. 4). On the contrary below $T_{\mathrm{N} 2}$, for instance at $6 \mathrm{~K}$, the magnetization increases rapidly around $\mu_{0} H=2 \mathrm{~T}$. This characteristic might be attributed to a metamagnetic like transition, probably due to a spinflip type of the Ce-ordered moments. The thermal dependence of the magnetization of the hydride $\mathrm{CeMnGeH}$ is characteristic of ferromagnetic, ferrimagnetic or canted systems (Fig. 3): (i) $M$ increases strongly at $T_{\mathrm{C}}=316(2) \mathrm{K}$, temperature deduced from the inflection point of the $M=f(T)$ curve; (ii) below $T_{\mathrm{C}}$, $M$ tends to saturate. Let us notice that no anomaly ascribable to the magnetic ordering of the Cemoments is detected here. This curve $M=f(T)$ is similar to that reported for $\mathrm{CeMn}_{2} \mathrm{Ge}_{2}$ where the Cemoments do not order down to $1.6 \mathrm{~K}$ [17].

The curves presenting the field dependence of the magnetization for $\mathrm{CeMnGeH}$ (Fig. 5) show the predominance of the antiferromagnetic interactions below $T_{C}=316(2) \mathrm{K}$ (above $\mu_{0} H=0.2 \mathrm{~T}, \mathrm{M}$ increases almost linearly with the applied magnetic field). The sharp initial rise in magnetization at low fields indicates a ferromagnetic component present for $T<T_{\mathrm{C}}$. These results suggest that CeMnGeH exhibits a canted magnetic ordering of the $\mathrm{Mn}$-sublattice. In other words, in the sequence $\mathrm{CeMnGe} \rightarrow \mathrm{CeMnGeH}$, the $\mathrm{Mn}-\mathrm{Mn}$ interactions existing in the $\left[\mathrm{Ce}_{4} \mathrm{Mn}_{4}\right]$ antiprisms seem to change from purely antiferromagnetic to a non-colinear antiferromagnetic structure. This transition could be correlated to a decrease of the $\mathrm{Mn}-\mathrm{Mn}$ intralayer spacings $(2.986 \rightarrow 2.928 \mathrm{~A}$ ) observed after the hydrogenation of CeMnGe. It is well known that in the CeMnSi and CeMnGe compounds, the decrease of the Mn-Mn interatomic distances induces an increase of the ferromagnetic feature [11]. Finally, it is noteworthy that these distances existing in $\mathrm{CeMnGeH}\left(2.928 \mathrm{~A}\right.$ ) are comparable with those observed in $\mathrm{CeMn}_{2} \mathrm{Ge}_{2}(2.932 \mathrm{~A}$ ) showing a conical magnetic structure [17].

\section{Conclusion}

The hydrogenation of the compounds $\mathrm{CeCoSi}, \mathrm{CeCoGe}$ and $\mathrm{CeMnGe}$ leads to an interesting change of the electronic state of cerium. The transition antiferromagnetic $\rightarrow$ spin fluctuation behavior evidenced by the hydrogenation of CeCoSi and $\mathrm{CeCoGe}$ and the disappearance of the magnetic transition associated to the Ce-moments in CeMnGeH suggest a lost of ordered magnetic moment on the Ce site during $\mathrm{H}$-insertion. This could result from the chemical effect of hydrogen which prevails over the unit cell expansion effect as explained in the case of $\mathrm{H}$-insertion into ternary silicide CeCoSi [13]. In order to solve this assumption, the electronic structure of $\mathrm{CeCoGe}, \mathrm{CeMnGe}$ and their hydrides are presently self consistently calculated within the local spin density functional (LSDF) theory.

\section{References}

[1] B. Chevalier, M. Pasturel, J.-L. Bobet, F. Weill, R. Decourt and J. Etourneau, J. Solid State Chem. 177 (2004), p. 752.

[2] B. Chevalier, J.-L. Bobet, E. Gaudin, M. Pasturel and J. Etourneau, J. Solid State Chem. 168 (2002), p. 28.

[3] B. Chevalier, M.L. Kahn, J.-L. Bobet, M. Pasturel and J. Etourneau, J. Phys.: Condens., Matter 14 (2002), p. L365.

[4] S.F. Matar, B. Chevalier, V. Eyert and J. Etourneau, Solid State Sci. 5 (2003), p. 1385.

[5] B. Chevalier, J.-L. Bobet, M. Pasturel, E. Bauer, F. Weill, R. Decourt and J. Etourneau, Chem. Mater. 15 (2003), p. 2181. 
[6] V.A. Yartys, B. Ouladdiaf, O. Isnard, O.Yu. Khyzhun and K.H.J. Buschow, J. Alloys Compd. 359 (2003), p. 62.

[7] B. Chevalier, M. Pasturel, J.-L. Bobet, J. Etourneau, O. Isnard, J. Sanchez Marcos and J. Rodriguez Fernandez, J. Magn. Magn. Mater. $272-276$ (2004) (Part 1), p. 576.

[8] B. Chevalier, E. Gaudin, F. Weill and J.-L. Bobet, Intermetallics 12 (2004), p. 437.

[9] J.-L. Bobet, S. Pechev, B. Chevalier and B. Darriet, J. Alloys Compd. 267 (1998), p. 136.

[10] J. Rodriguez-Carvajal, Physica B 192 (1993), p. 55.

[11] R. Welter, G. Venturini, E. Ressouche and B. Malaman, J. Alloys Compd. 228 (1995), p. 59.

[12] R. Welter, G. Venturini and B. Malaman, J. Alloys Compd. 189 (1992), p. 49.

[13] B. Chevalier and S.F. Matar, Phys. Rev. B 70 (2004), p. 174408.

[14] R. Welter, G. Venturini, B. Malaman and E. Ressouche, J. Alloys Compd. 201 (1993), p. 191.

[15] J. Lawrence, Phys. Rev. B 20 (1979), p. 3770.

[16] W.H. Lee, H.C. Ku and R.N. Shelton, Phys. Rev. B 36 (1987), p. 5739.

[17] R. Welter, G. Venturini, E. Ressouche and B. Malaman, J. Alloys Compd. 218 (1995), p. 204.

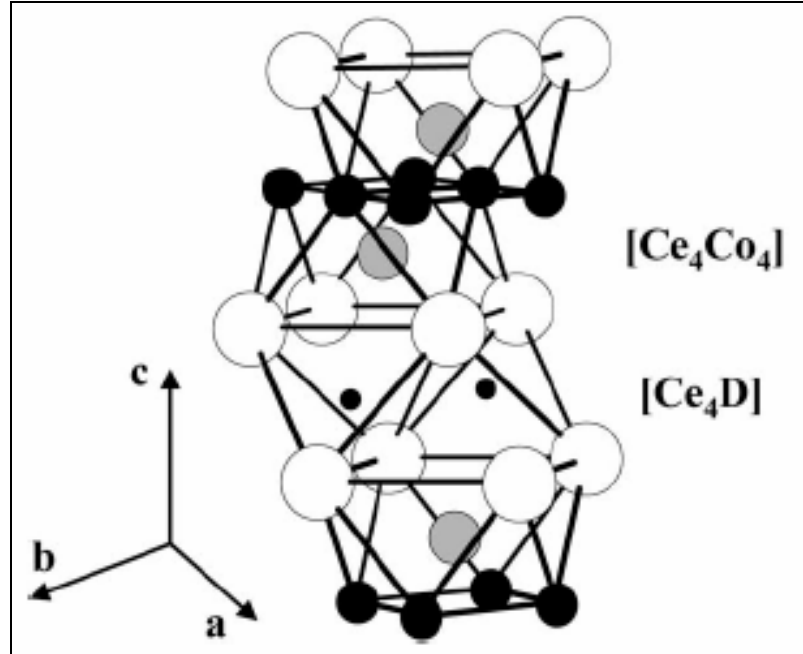

Fig. 1. Crystal structure of CeCoGeD. (Ce, Co, Ge and D atoms are, respectively, represented by white large, black medium, grey medium and black small circles). 
Table 1

Crystallographic data relative to the ternary compounds $\mathrm{CeMnGe}, \mathrm{CeFeSi}$ and $\mathrm{CeCoX}(\mathrm{X}=\mathrm{Si}$ and $\mathrm{Ge})$ and their hydrides

\begin{tabular}{|c|c|c|c|c|c|c|c|}
\hline \multirow[t]{2}{*}{ Compound } & \multicolumn{3}{|c|}{ Unit cell parameters } & \multirow[t]{2}{*}{$\Delta a / a(\%)$} & \multirow[t]{2}{*}{$\Delta c / c(\%)$} & \multirow[t]{2}{*}{$\Delta V / V(\%)$} & \multirow[t]{2}{*}{ Ref. } \\
\hline & $a(\AA)$ & $c(\AA)$ & $V\left(\AA^{3}\right)$ & & & & \\
\hline $\mathrm{CeMnGe}$ & $4.235(3)$ & $7.386(6)$ & $132.5(3)$ & & & & [11] \\
\hline $\mathrm{CeMnGe}$ & $4.2232(2)$ & $7.3513(4)$ & $131.1(3)$ & & & & a \\
\hline $\mathrm{CeMnGeH}_{1.1(1)}$ & $4.1408(2)$ & $7.9401(3)$ & $136.1(3)$ & -2.0 & 8.0 & 3.8 & a \\
\hline $\mathrm{CeFeSi}$ & $4.086(2)$ & $6.788(3)$ & $113.3(3)$ & & & & [12] \\
\hline $\mathrm{CeFeSiH}_{1 \circ(1)}$ & $3.994(2)$ & $7.810(4)$ & $124.6(3)$ & -2.3 & 15.1 & 10.0 & a \\
\hline $\mathrm{CeCaSi}$ & $4.041(2)$ & $6.990(2)$ & $114.1(3)$ & & & & [13] \\
\hline $\mathrm{CeCoSiH}_{1 \propto(1)}$ & $3.955(2)$ & $7.861(3)$ & $123.0(3)$ & -2.1 & 12.5 & 7.8 & [13] \\
\hline $\mathrm{CeCoGe}$ & $4.170(2)$ & $6.865(2)$ & $119.4(3)$ & & & & [9] \\
\hline $\mathrm{CeCoGeH}_{1.97(9)}$ & $4.040(2)$ & $7.735(4)$ & $126.2(4)$ & -3.1 & 12.7 & 5.7 & [9] \\
\hline
\end{tabular}

a This work.

Table 2

Result of the neutron refinement for $\mathrm{CeCoGe}$ and $\mathrm{CeCoGeD}$ at room temperature (space group P4/mmm, no. 129)

\begin{tabular}{|c|c|c|c|c|c|}
\hline Atom & Site & $x$ & y & $z$ & $B\left(10^{-2} \AA^{2}\right)$ \\
\hline \multicolumn{6}{|c|}{$\overline{\mathrm{CeCoGe}}$} \\
\hline $\mathrm{Ce}$ & $2 c$ & $1 / 4$ & $1 / 4$ & $0.6812(14)$ & $0.4(1)$ \\
\hline Co & $2 a$ & $3 / 4$ & $1 / 4$ & 0 & $0.3(2)$ \\
\hline $\mathrm{Ge}$ & $2 c$ & $1 / 4$ & $1 / 4$ & $0.1924(8)$ & $0.17(8)$ \\
\hline \multicolumn{6}{|c|}{$\begin{array}{l}a=4.190(1) \AA \text { and } c=6.872(2) \AA ; \\
C e C o G e D\end{array}$} \\
\hline $\mathrm{Ce}$ & $2 c$ & $1 / 4$ & $1 / 4$ & $0.6735(14)$ & $0.2(2)$ \\
\hline Co & $2 a$ & $3 / 4$ & $1 / 4$ & 0 & $0.8(2)$ \\
\hline $\mathrm{Ge}$ & $2 c$ & $1 / 4$ & $1 / 4$ & $0.1632(8)$ & $0.3(1)$ \\
\hline D & $2 \mathrm{~b}$ & $1 / 4$ & $3 / 4$ & $1 / 2$ & $1.7(2)$ \\
\hline \multicolumn{6}{|c|}{$a=4.044(1) \AA$ and $c=7.746(2) \AA ; R_{\mathrm{B}}=9 \%, R_{\mathrm{p}}=3.5 \%$ and $R_{\mathrm{mp}}=5.13 \%$} \\
\hline
\end{tabular}




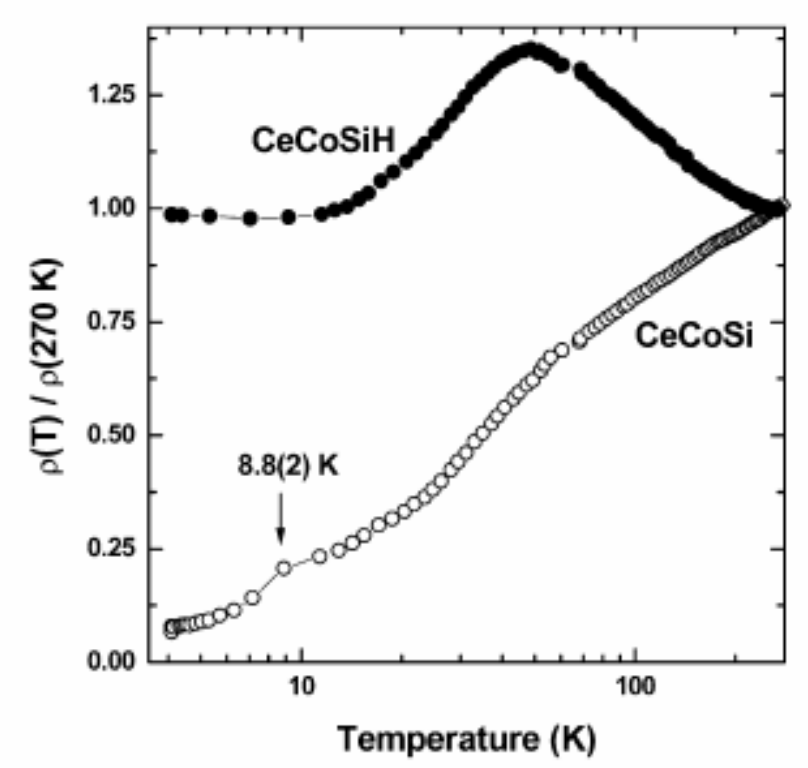

Fig. 2. Reduced electrical resistivity as a function of $\log T$ for $\mathrm{CeCoSi}$ and its hydride.

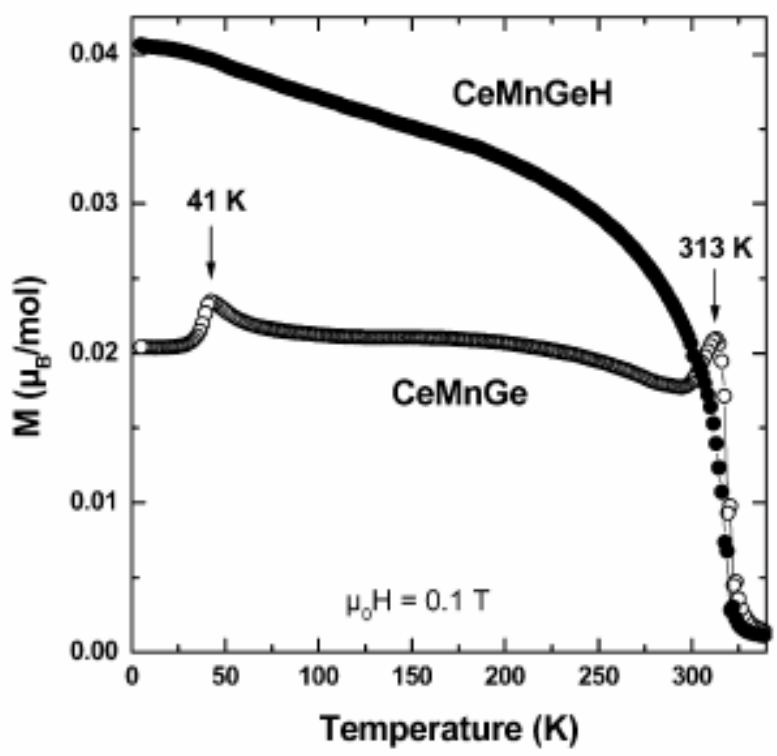

Fig. 3. Temperature dependence of the magnetization, measured in the field $\mu_{0} H=0.1 \mathrm{~T}$, for $\mathrm{CeMnGe}$ and its hydride.

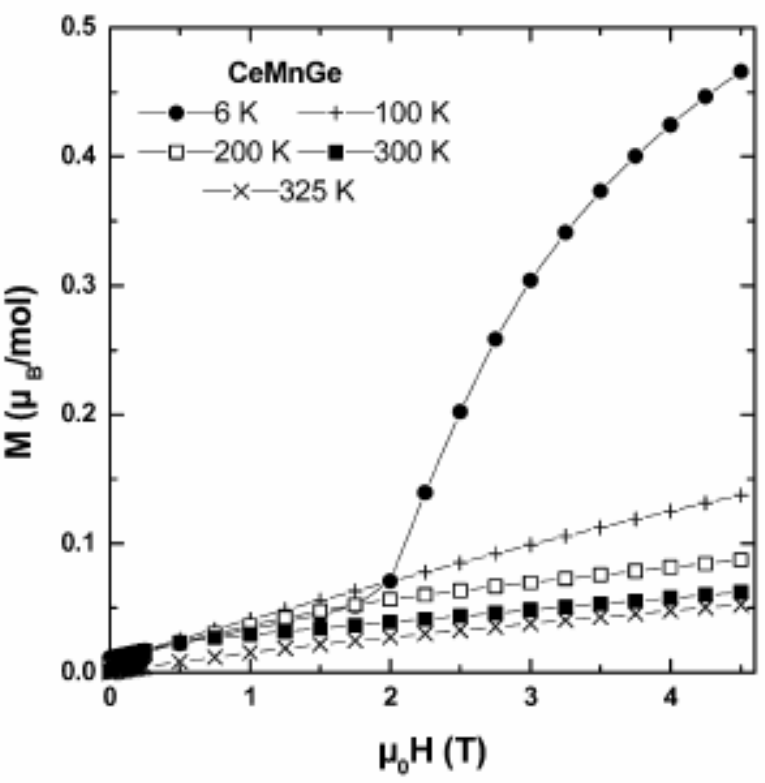

Fig. 4. Field dependence of the magnetization for $\mathrm{CeMnGe}$ at selected temperatures. 


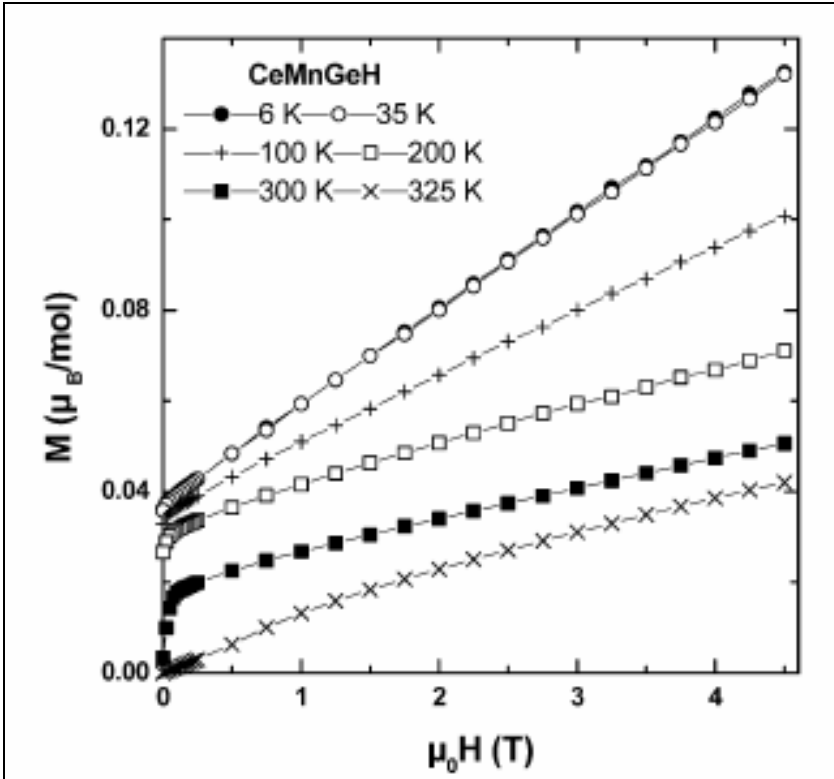

Fig. 5. Field dependence of the magnetization for $\mathrm{CeMnGeH}$ at selected temperatures. 